\title{
Resolução de problemas matemáticos como alternativa para o desenvolvimento cerebral e fortalecimento da saúde mental: uma revisão bibliográfica
}

\author{
Solving mathematical problems as an alternative for brain development and strengthening \\ mental health: a literature review
}
Resolver problemas matemáticos como una alternativa para el desarrollo del cerebro y fortalecer la salud mental: una revisión de la literatura

Sheila Chaves Batista do Nascimento ${ }^{1 *}$, Joccitiel Dias da Silva ${ }^{1}$.

\begin{abstract}
RESUMO
Objetivo: Objetiva-se nesta revisão propor a utilização de problemas matemáticos como forma de fortalecer a saúde mental e desenvolver o cérebro humano. Revisão Bibliográfica: A resolução de problemas vem aparecendo de forma inovadora como metodologia para o ensino matemático em diversas pesquisas educacionais. A pesquisa em questão, aborda também a utilidade da resolução de problemas matemáticos para o desenvolvimento cerebral e aumento da qualidade de vida através do fortalecimento da saúde mental. Exercitar o cérebro e estar em constante movimento, faz com que o corpo físico mantenha suas funções exercitadas e saudáveis, assunto que é objetivo de inúmeras pesquisas científicas e colaborações entre as áreas da saúde e da educação. A finalidade central de inserir a resolução matemática nos cuidados com a saúde mental, é instruir novos métodos e ampliar a margem de estudos nessa nova estratégia. Considerações finais: $O$ estudo se baseia na metodologia revisional para reunir todos os conhecimentos já existentes na área da saúde mental correlacionado com educação e como podem interagir ambas as áreas a fim de promover o fortalecimento da mente.
\end{abstract}

Palavras-chave: Educação e saúde, Saúde mental, Saúde do cérebro.

\begin{abstract}
Objective: The aim of this review is to propose the use of mathematical problems as a way to strengthen mental health and develop the human brain. Bibliographic Review: Problem solving has been appearing in an innovative way as a methodology for mathematical teaching in several educational researches. The research in question also addresses the usefulness of solving mathematical problems for brain development and increasing quality of life through strengthening mental health. Exercising the brain and being in constant movement, makes the physical body keep its functions exercised and healthy, a subject that is the objective of numerous scientific researches and collaborations between the areas of health and education. The central purpose of inserting mathematical resolution in mental health care is to instruct new methods and expand the scope of studies in this new strategy. Final considerations: The study is based on the revision methodology to gather all the knowledge already existing in the area of mental health related to education and how they can interact both areas in order to promote the strengthening of the mind.
\end{abstract}

Keywords: Education and health, Mental health, Brain health.

${ }^{1}$ Faculdade Vale do Cricaré (FVC), São Mateus - ES. *E-mail: sheilaartigo2020@gmail.com.

SUBMETIDO EM: 6/2020

ACEITO EM: 7/2020

PUBLICADO EM: 10/2020

REAS/EJCH | Vol.12(10) | e4397 | DOI: https://doi.org/10.25248/reas.e4397.2020 Página 1 de 7 


\section{RESUMEN}

Objetivo: El objetivo de esta revisión es proponer el uso de problemas matemáticos como una forma de fortalecer la salud mental y desarrollar el cerebro humano. Revisión bibliográfica: la resolución de problemas ha aparecido de manera innovadora como metodología para la enseñanza matemática en varias investigaciones educativas. La investigación en cuestión también aborda la utilidad de resolver problemas matemáticos para el desarrollo del cerebro y aumentar la calidad de vida al fortalecer la salud mental. Ejercer el cerebro y estar en constante movimiento, hace que el cuerpo físico mantenga sus funciones ejercitadas y saludables, un tema que es el objetivo de numerosas investigaciones científicas y colaboraciones entre las áreas de salud y educación. El propósito central de insertar la resolución matemática en la atención de la salud mental es instruir nuevos métodos y ampliar el alcance de los estudios en esta nueva estrategia. Consideraciones finales: El estudio se basa en la metodología de revisión para recopilar todo el conocimiento ya existente en el área de la salud mental correlacionado con la educación y cómo pueden interactuar ambas áreas para promover el fortalecimiento de la mente.

Palabras clave: Educación y salud, Salud mental, Salud del cerebro.

\section{INTRODUÇÃO}

Embora seja foco de um grande número de estudos que visam entender este problema, até o presente momento os indicadores de qualidade da educação básica apontam que o Sistema Educacional Brasileiro (SEB) necessita de melhorias. Segundo dados do Programa Internacional de Avaliação de Alunos (PISA), uma prova que é aplicada em 70 países. O Brasil ficou na $66^{a}$ colocação em matemática. A pontuação dos alunos brasileiros caiu de 391 para 377. Almejou-se uma solução que fosse eficaz em diversos aspectos para esta problemática. Entretanto, observou-se a necessidade de estudar cada situação e os indivíduos pertencentes a elas a fim de superar os paradigmas referentes à educação matemática, momento no qual a etnomatemática aparece como auxílio no processo de aprendizagem (PASSOS CLB e NACARATO AM, 2018).

Com base em vários métodos e materiais de referência, e usando uma variedade de métodos, são estudados os fatores que influenciam a composição do campo brasileiro de ensino de ciências e matemática e a origem e as características das pesquisas realizadas nesse campo. A grande quantidade de conhecimentos acumulados nesse campo é resultado do trabalho de matemáticos, físicos, químicos, biólogos e outros profissionais, que realizaram pesquisas acadêmicas sobre essas questões e foram sistematicamente mapeados e mapeados por muitos autores (NARDI R, 2015).

Segundo Valente WR (2008), a Matemática na Educação Básica brasileira é uma disciplina em que se observa que um elevado quantitativo de alunos apresenta, durante o processo educativo, um rendimento qualitativo e quantitativo insatisfatório. As causas são diversas. Porém, o autor destaca que a forma como os conteúdos curriculares desta disciplina está sendo ensinada na mediação pedagógica tem sido crucial para o baixo rendimento. Ele expõe ainda que os procedimentos metodológicos colaboram para os baixos níveis de aprendizagem. De tal forma, objetivar práticas de desenvolvimento mental para promover saúde cerebral, requer antes uma elevação e melhor controle da qualidade da educação brasileira.

Propor uma reflexão acerca de problemas matemáticos coadunando tal conhecimento com saúde mental, é um desafio para qualquer pesquisador. Entretanto, as áreas que dissecam os conhecimentos na área da saúde, dependem indispensavelmente do conhecimento das áreas de exatas, tanto para calcular uma fórmula medicamentosa, quanto para adentrar à fórmulas complexas de estruturas celulares entre outras situações. Os problemas matemáticos não só dependem da atenção redobrada, como quando posto a desafiar quem vai se dedicar a sua resolução requer uma dose maior de paciência, haja vista que os resultados muitas das vezes já são conhecidos, e o desafio está em alcança-lo através de uma fórmula matemática (MIRANDA PR e GAZIRE ES, 2012). 
Atualmente, no Brasil, existem muitos problemas com a educação no sistema público de ensino, como o abandono escolar e até crianças que não sabem ler, escrever e que não conseguem passar na escola. Os professores reclamam da falta de concentração, falta de interesse, violência e disciplina dos alunos, olhando apenas o externo e não sabendo identificar o que existe por detrás da falta de atenção e dedicação (MAZER SM, et al., 2009).

O histórico das disciplinas da área de exatas apresenta uma grande expectativa quanto a aptidão do discente, entretanto, nada se analisa quando as possibilidades de inserções diferentes dos métodos de ensino. Os profissionais da educação, especificamente os da área citada, não recebem adequadamente os preparos necessários para explorar as individualidades do aluno. Outro fator que impossibilita uma abordagem mais profunda nas singularidades, é a ausência de profissionais suficientes nas salas de aula além do pouco preparo em questões referentes a democratização do ensino. A escola, mesmo que tenha ampliado a linha de acesso, pouco trabalhou questões individuais na busca por preencher os espaços causados pela desigualdade social (WERLE FOC, 2014).

Questões como saúde mental, fracasso escolar e democratização do ensino, estão todas interligadas. Estudos de áreas completamente distintas podem se completar e garantir dados satisfatórios se tratando dos objetivos desta pesquisa. O objetivo desta revisão é apresentar estudos que fomentem a utilização de problemas matemáticos como alternativa ao desenvolvimento cerebral e promoção da saúde mental, de forma a garantir que novas práticas sejam implementadas no sistema educacional brasileiro, com intenção de alcançar os alunos que possuem maiores dificuldades quando se tratam de ciências exatas. É também objetivo deste estudo, explorar a realidade do abandono escolar por meio do fracasso do aluno, que por muitas vezes pode se tratar de falta de sensibilidade do ambiente de estudo.

\section{REVISÃO BIBLIOGRÁFICA}

A matemática tem sido e continuará sendo um campo fundamental de todos os sistemas educacionais. Por centenas de anos, essa é uma ciência muito antiga que se tornou parte da disciplina escolar. Portanto, durante o longo ano letivo, eles devem ser obrigatoriamente ensinados e obrigados a desempenhar um papel importante nas escolhas sociais. É também considerado como uma linguagem absoluta, um padrão impecável e a chave para o progresso. Outras ciências nos permitem entender os mistérios dos seres humanos, da natureza, do mundo e do universo, e eles dependem principalmente da matemática, no entanto, a importância deste ponto está longe disso (OLIVEIRA MF, et al., 2015).

A saúde mental é um dos campos mais complexos de todas as ciências já estudadas. Requer não só muita atenção da parte dos seus pesquisadores, mas extremo engajamento na finalidade de alcançar os resultados que podem influenciar em grande quantidade na qualidade de vida do ser humano. Um ambiente saudável estará sempre atrelado ao quanto ele influencia bem no desenvolvimento e na preservação da mente. $O$ adoecimento está quase sempre ligado, no seu princípio, ao estresse ou a condições desumanas de convivência. Nota-se com precisão o despreparo do ser humano com as ações que alcançam diretamente o próximo, já que a disseminação do egocentrismo influencia as sociedades desde que o ser humano passou a demarcar posse e tornar isso mais importante que satisfazer as necessidades sociais do coletivo (GARCIA JM, 2016).

A reforma psiquiátrica trouxe uma nova maneira de olhar para a saúde mental e delineia novas diretrizes para o tratamento e a reintegração dos indivíduos na sociedade: garantindo-lhes cidadania plena e promovendo os meios que lhes permitem administrar suas vidas. Portanto, é necessário apoiar essas pessoas com base no respeito à singularidade de cada ser. No novo plano, uma nova forma de trabalho emergiu em algumas instituições: a prática de vários trabalhos coletivos dentro da instituição, que está além do escopo do tratamento intramural (LANZA LD e DETONI AR, 2013).

Dessas novas maneiras, surgiram novas práticas. As práticas utilizadas na busca de restaurar a saúde mental ou abrir novas portas de tratamento, a realização de cálculos, que é um exercício que promove o desenvolvimento do cérebro, surge como opção. À partir que o cérebro humano se desenvolve e tem áreas pouco conhecidas sendo ativadas, as soluções para os problemas antes não tratados, podem surgir (LANZA LD e DETONI AR, 2013). 
A aprendizagem está pautada sob muitos aspectos, ela envolve muitos campos e muitas linhas ainda não exploradas. Explorar o cérebro humano sob uma perspectiva matemática pode parecer sem sentido, mas aos olhos científicos e profissionais não se trata de nenhum desafio. O cérebro humano ainda é muito pouco explorado, pouco se sabe com relação à boa parte da sua funcionalidade. Do pouco que se conhece do cérebro humano, sabe-se que o seu pleno exercício garante a longevidade das suas funcionalidades de maneira mais saudável, e o exercício promove uma ampliação nas suas funções e estende a área utilizável do mesmo. Várias civilizações antigas apresentaram desde os mais simples métodos de contagem já existentes, aos mais desafiadores e problemáticos cálculos e bases exatas, que na nossa civilização atual dependeriam de um conhecimento aprofundados da engenharia civil (CARDOSO TSG e MUSZKAT M, 2018).

Quando pouco se trabalha as habilidades do aluno e classifica-se o conhecimento não convencional desprezível dentro do ambiente escolar, isso torna o discente um propenso dado a mais nos números da evasão escolar. O ensino, por vezes, pode ser insensível, assim como o ambiente em que ele é disseminado. Não priorizar as necessidades comungando-as com as dificuldades e explorando os potenciais, é desrespeitar a individualidade humana, que de tão importante possui defesa nas mais importantes legislações do planeta, como também potencializa a perda de grandes mentes e genialidades ímpares para a ciência. Quando o docente não compreende a linha de pensamento do aluno, não significa que ele não apresentou algo com sentido ou que não possa ser explorado. Seria coeso considerar que somente o professor possui o conhecimento? Ou que o aluno não poderia desenvolver através de práticas, que por vezes, seriam basicamente um pedido de socorro ou uma pista para que ele também possa ser alcançado educacionalmente. (TARTUCE GLBP, et al., 2018).

O fracasso escolar faz parte da história da educação pública no Brasil, mas, nas últimas décadas, esse assunto tem recebido mais atenção, principalmente devido a avaliações externas. Quando a escola é considerada o espaço responsável pelo ensino de conhecimento científico aos alunos, confirmamos a teoria histórica e cultural, cujo conteúdo é o matemático, e muitas pessoas pensam que a matemática é uma das áreas de conhecimento mais difíceis de entender. Não apenas os alunos que estão relacionados ao conteúdo têm dificuldades, mas também os professores que frequentemente têm dificuldades no ensino de matemática. Devido a isso, o fracasso escolar é um dos principais causadores da evasão escolar, e importa tratar desse assunto na pesquisa, pois o emprego errado da matemática e a ausência de sensibilidade para compreender as limitações do aluno, acabam se tornando empecilho para melhorar os níveis da educação (WEBER TM, et al., 2013).

\section{Problemas matemáticos e o desenvolvimento cerebral}

Estudos relacionados a neurociência avançaram nos últimos anos, isso permitiu que este campo de estudo alcançasse a área da educação. Buscar aparato científico na finalidade de introduzir novas técnicas é uma forma de justificar a qualidade daquilo que está sendo investido. Para áreas como saúde e educação, é imprescindível um parecer técnico e a intenção do pesquisador ser inclinada a objetivos nobres. Entretanto, não se trata de estudos superficiais ou averiguações apenas por imagem ou teóricas, mas um campo quase que vazio de dados e profundo no que tange a análise do cérebro humano, principalmente análises momentâneas, quando o estudado está em plena atividade cerebral. Aparelhos como neurotransmissores e neuromoduladores são utilizados, o que amplia a capacidade da pesquisa, mas delimita seu campo de desenvolvimento (FERNANDES CT, et al., 2015).

O cérebro depende de atividades e exercícios, não somente exercícios físicos, que já é de grande importância para manter o corpo saudável além de ativar a circulação de informações neurais, mas também de atividades intelectuais, que desafiam o ser a colocar o pensamento em ação. Uma criança está em fase desenvolvimento e o cérebro certamente não alcançou a quantidade de áreas ativas que alcança em um adulto saudável, isso faz com que seja necessário estimular o pensamento e instruir atividades mentais para que a criança tenha um desenvolvimento, além de saudável, mais preciso. A finalidade do desenvolvimento mental estará sempre atrelada a necessária capacidade de lidar com dificuldades e situações não comuns. Um exemplo muito exposto no meio científico é o desenvolvimento da linguagem, que é indispensável para a convivência social (BUCHWEITZ A, 2016). 
Das inúmeras atividades que colaboram para desenvolver o cérebro humano, é importante atentar para a utilização de cálculos matemáticos. Toda atividade que o corpo humano produz, gera imediatamente informações que são levadas ao cérebro, que é a máquina que controla e comanda o corpo humano. A partir do momento que as informações são enviadas ao cérebro na resolução de algo problemático, este trabalhará para alcançar a resolução deste problema, buscando informações úteis que levem ao resultado ou que levem a caminhos que possam o possibilitar. A ativação das áreas do cérebro permitem uma ampliação do campo utilizado por ele, isso faz com que em novas situações o cérebro já saiba como agir, e tornando aquela área utilizada útil para a resolução de outras situações que antes não eram permitidas, devido a ausência de atividade cerebral na área específica (CARVALHO FAH, 2010).

A finalidade de se alcançar o desenvolvimento cerebral em uma criança é aprimorar a convivência social e garantir uma melhor maneira de se alcançar o aprendizado. A importância da matemática visual para se alcançar o aprendizado em crianças é o fortalecimento dos meios de processar as informações, e áreas que respondem pelo controle da atenção. As diferenças intelectuais são necessariamente tocadas e os estudos aprofundados têm por finalidade reduzi-las também, ainda se tratando das áreas das ciências exatas, por se tratar muitas das vezes de um desafio a parte para boa parte dos alunos, buscar torna-las menos complexas ou tornar o ser humano mais resolutivo.

A criança quando recebe comandos uma primeira vez, não consegue identificar como proceder, apenas o exercício constante de aprendizado vai fazer com que ela entenda, da mesma forma acontecerá na resolução de problemas matemáticos (OLIVEIRA MF, et al., 2015).

Em crianças com necessidades especiais, a matemática também representa um desafio, só que muito maior, principalmente para aqueles com deficiências mentais. Para crianças com deficiências físicas, mas que gozam do completo funcionamento do cérebro, as dificuldades podem ocorrer desde as dificuldades em escrever, até no desconforto que algumas pressões podem causar. Mas pesquisas apontam que o uso de da matemática no tratamento de doenças mentais, como a epilepsia por exemplo, pode colaborar para ampliar o autocontrole, o que reduziria os efeitos das crises. Há um entendimento entre diferentes estudos e pesquisas no que faz relação positiva entre epilepsia e dificuldades em aprender, principalmente quando se trata do domínio da matemática. A chave para a resolução de tal situação não poderia estar atrelada a própria dificuldade? (HAZIN I, et al., 2009).

Existem inúmeras maneiras de utilizar a matemática para resolver problemas e situações relacionadas ao desenvolvimento cerebral, de tal forma a colaborar com o desenvolvimento intelectual do aluno, mas também de todo o conjunto social que o rodeia. Desde que existem teorias sobre a evolução humana, como a teoria da evolução de Charles Darwin, há percepções, tanto em pesquisas físicas com fósseis e restos humanos de milênios atrás, e também conhecimentos teóricos baseados nas evidências coletadas, que o cérebro do ser humano está em constante expansão, principalmente no que retrata seu tamanho.

É notado que essas teorias indicam que o ser humano ainda evolui na linha do tempo, o que reforça a teoria de que a evolução também parte do desenvolvimento intelectual e da utilização do cérebro para alcançar resultados novos em campos ainda desconhecidos (CELERI EHRV, et al., 2010).

\section{Fracasso escolar e saúde mental}

O fracasso escolar é um assunto amplamente discutido no meio educacional. Diante disso, autores e pesquisadores como alertam que o retrocesso vislumbrado na atualidade do processo educacional e a busca por explicações sobre o fracasso escolar já deveriam ter sido superados, pois é possível perceber retrocessos e avanços nesse sentido. É comum perceber muitos pontos que influem na questão do fracasso escolar, sejam eles culturais ou não, podem desencadear uma onda de situações complexas.

O aluno quando não está ambientado ou quando é submetido a um ambiente hostil, certamente terá mais dificuldades de desenvolver o lado crítico e consequentemente, está menos fadado a buscar ajuda nos momentos de dificuldade. Situações complexas como essas, podem potencializar os efeitos da evasão escolar, ou agirem como próprio agente causador desse distúrbio social (PAULA FS e TFOUNI LV, 2009). 
A ligação do fracasso escolar com o adoecimento mental do aluno é muito profunda e objeto de estudos constantes, já que a partir do adoecimento da mente que se iniciam inúmeros processos de adoecimento do corpo físico. O aluno quando não consegue atingir seus objetivos escolares ou quando não possui aparato suficiente para estar adequado ao que se considera sucesso escolar, ele recebe uma carga altíssima de preconceitos emitidos pela sociedade, que consequentemente transmite tais pesos as famílias e que são todos despejados no discente através de expectativas frustradas. Identificar a raiz do problema abre inúmeras portas para buscar resolutividades, entretanto, não se caracteriza como uma verdade real, já que se pode identificar ao longo da história da educação, erros e acertos nas abordagens das suas teorias. Entender o problema e construir reações positivas que permitam ir mais a fundo nas suas investigações, é uma abordagem mais coesa quando se trata das ciências educacionais (CID MFB, et al., 2019).

A palavra fracasso possui uma carga muito pesada de preconceitos, já que em uma sociedade extremamente capitalista, onde a competição é incentivada desde tenra idade, fracassar significa não ser suficiente para tal. Fracassar na escola seria o primeiro passo para fracassar na vida. Entretanto, o significado da palavra fracasso para a questão do fracasso escolar, tem um tom mais apaziguador, que leva ao entendimento de que soluções estão sendo buscadas e a culpabilização do aluno está sendo reduzida.

É comum ouvir pessoas reproduzindo frases indicando a culpa a professores ou alunos quando surgem problemas na educação, mas a raiz do problema está sempre ligada a sociedade e como ela não se permite participar do processo escolar. A escola busca refletir as ideias da sociedade e ambientar o prédio para melhor acolher e permitir que todos possam se sentir bem (SILVA CS e COSTA BLD, 2016).

Para uma criança, ouvir uma palavra negativa ou se sentir repreendida, pode significar uma infinidade de coisas. Quando a criança recebe um não quanto ao seu direito a aprender, mesmo que não reproduzido diretamente, é uma informação que vai pesar e influir em uma série de situações. Dentro da sala de aula, não saber significa fracasso, esta é a realidade. A disciplina de matemática apresenta uma infinidade de situações que afugenta os alunos, principalmente quando se dá a dificuldade na resolução dos cálculos.

Um aluno que não tem aptidão para disciplinas exatas, pode sofrer uma abordagem hostil da família, já que se normalizou a ideia do "se estudar consegue", e nem sempre é assim. Da mesma forma, essa hostilidade também é comum quando não se tem aptidão em outras disciplinas, e a culpa estará sempre dispensada ao aluno e quase nunca aos métodos de aplicação (PEZZI FAS e MARIN AH, 2017).

Nesse sentido, alguns autores e pesquisadores ressaltam que uma boa base na educação é primordial, pois os sistemas de ensino devem atender às diversidades das necessidades presentes nas escolas. $\mathrm{E}$, para isso, é crucial identificar onde se localizam as inadaptações à aprendizagem, orientando para que o aluno possa descobrir a própria maneira de aprender, considerando como ponto indispensável o modo particular de cada um se relacionar com a organização da aprendizagem escolar e os conhecimentos prévios que esses estudantes possuem. Os diversos fatores que impactam no baixo rendimento escolar são objetos de estudos de muitos pesquisadores, pois o termo é cada vez mais frequente no ambiente escolar (MELCHIOR MC, 2014).

Diante desse cenário, a resolução de problemas é considerada uma fonte motivadora para a compreensão e a utilização do conhecimento matemático de forma adequada e eficaz. a avaliação diagnóstica escolar possibilitará a produção de uma gama de análise bem ampla e significativa em relação ao conteúdo do currículo escolar que estão defasados, o que permite a busca de meios pelos quais seja possível propiciar um processo de aprendizado significativo, reduzindo o índice de reprovação.

Observa-se que o foco voltado para a resolução de problemas matemáticos de forma inovadora tende a suprir a carência observada nesta disciplina. E produzir métodos que colaborem para que sejam superadas as dificuldades dos alunos, é um fator forte da defesa de que um ambiente escolar saudável se constrói com atenção e não com hostilidades, de maneira a preservar e garantir o desenvolvimento escolar através de práticas humanizadas (LUCKESI CC, 2011). 


\section{CONSIDERAÇÕES FINAIS}

Conclui-se, portanto, sabendo que é possível comunicar inúmeras ciências na busca pela resolução de problemas complexos. A utilização da matemática como forma de alcançar desenvolvimento cerebral, e os métodos não hostis através da aplicação das disciplinas, na intenção de garantir e preservar a saúde mental do aluno, são práticas aprimoráveis e que merecem uma atenção maior dos cientistas das teorias da educação. A ciência matemática deve ser utilizada como método de desenvolvimento cerebral, e suas práticas aprimoradas na escola, com a finalidade de compreender o problema do aluno quando este não alcança o conhecimento, evitando que o mesmo se afaste da escola e se torne um caso mais complexo. É com base na reunião da bibliografia que foi possível encontrar essas prováveis saídas para o desenvolvimento cerebral através da inserção de cálculos.

\section{REFERÊNCIAS}

1. BUCHWEITZ A. Desenvolvimento da linguagem e da leitura no cérebro atualmente: neuromarcadores e o caso de predição. Jornal de Pediatria, 2016; 92(3): 8-13.

2. CARDOSO TSG, MUSZKAT M. Aspectos neurocientíficos da aprendizagem matemática: explorando as estruturas cognitivas inatas do cérebro. Revista Psicopedagogia, 2018; 35(105): 73-81.

3. CARVALHO FAH. Neurociências e educação: uma articulação necessária na formação docente. Trabalho, Educação e Saúde, 2010; 8(3): 537-550.

4. CELERI EHRV, et al. Charles Darwin: um observador do desenvolvimento humano. Revista Latinoamericana de Psicopatologia Fundamental, 2010; 13(4): 558-576.

5. CID MFB, et al. Saúde mental infantil e contexto escolar: as percepções dos educadores. Pro-Posiçõe; 1(30): 3-24.

6. CRUZ V. Desenvolvimento cognitivo e aprendizagem da matemática. Análise Psicológica, 2014; 32(1): $127-132$.

7. FERNANDES CT, et al. Possibilidades de aprendizagem: reflexões sobre neurociência do aprendizado, motricidade e dificuldades de aprendizagem em cálculo em escolares entre sete e 12 anos. Ciência \& Educação (Bauru), 2015; 21(2): 395-416.

8. GARCIA JM. Saúde Mental na Escola: O que os Educadores Devem Saber. Psico-USF, 2016; 21 (2): $423-425$.

9. HAZIN I, et al. Abordagem neuropsicológica da aprendizagem matemática escolar em crianças com necessidades educacionais especiais. Cadernos de Psicopedagogia, 2009; 7(13): 1-25.

10. LANZA LD, DETONI AR. Atividades matemáticas e saúde mental. Encontro Nacional de Educação Matemática, 2013; 1(1): 1-10.

11. LUCKESI CC. Avaliação da aprendizagem escolar: estudos e proposições. 22a ed. São Paulo: Cortez, 2011.

12. MAZER SM, et al. Dificuldades de aprendizagem: revisão de literatura sobre os fatores de risco associados. Psicologia da Educação, 2009; 28(1): 7-21.

13. MELCHIOR MC. O sucesso escolar através da avaliação e da recuperação. Porto Alegre: Premier, 2014.

14. MIRANDA PR, GAZIRE ES. Saúde e números: uma parceria de sucesso. Bolema: Boletim de Educação Matemática, 2012; 26(42): 609-626.

15. NARDI R. A pesquisa em ensino de Ciências e Matemática no Brasil. Ciência \& Educação (Bauru), $2015 ; 21$ (2): 1-7.

16. OLIVEIRA MF, et al. Condicionantes da aprendizagem da matemática: uma revisão sistêmica da literatura. Educ. Pesqui, 2015; 41(4): 1023-1037.

17. PASSOS CLB, NACARATO AM. Trajetória e perspectivas para o ensino de Matemática nos anos iniciais. Estudos Avançados, 2018; 32(94): 119-135.

18. PAULA FS, TFOUNI LV. A persistência do fracasso escolar: desigualdade e ideologia. Revista Brasileira de Orientação Profissional, 2009; 10(2): 117-127.

19. PEZZI FAZ, MARIN AH. Fracasso escolar na educação básica: revisão sistemática da literatura. Temas em Psicologia, 2017; 25(1): 1-15.

20. SILVA CS, COSTA BLD. Opressão nas escolas: o bullying entre estudantes do ensino básico. Cadernos de Pesquisa, 2016; 46(161): 638-663.

21. TARTUCE GLBP, et al. Desafios do ensino médio no Brasil: iniciativas das secretarias de educação. Cadernos de Pesquisa, 2018; 48(168): 478-504.

22. VALENTE WR. Quem somos nós, professores de matemática? Cad. Cedes, 2008; 28(74): 11-23.

23. WEBER TM, et al. Educação matemática escolar: o fracasso do aluno ou do sistema? XI Congresso Nacional de Educação - EDUCARE, 2013: 25774-25782.

24. WERLE FOC. Panorama das políticas públicas na educação brasileira: uma análise das avaliações externas de sistemas de ensino. Revista Lusófona de Educação, 2014; 27(1): 159-179. 